Christiane Högermann, Mely Kiyak \& Konstanze Neubauer

Gärten des Jahres (2017) - Die 50 schönsten Privatgärten

Callwey Verlag, München, 2017, 272 Seiten, 238 Abb., ISBN 978-3-7667-2252-2, 59,95 Euro

Diese eindrucksvolle Foto-Text-Dokumentation führt den Leser, oder besser „Zuschauer“, mit über 200, zum Teil doppelseitigen Panoramabildern durch die 50 für 2017 im wahrsten Sinne des Worts (im Wettbewerb „Gärten des Jahres“) ausgezeichneten, ausschließlich von professionellen Gestaltern angelegten Gärten in ganz Deutschland und einigen Schweizer Kantonen. Bereits beim Durchblättern lassen diese kleinen Paradiese den Leser in exklusive botanisch-gartenarchitektonische Welten eintauchen und spiegeln auch die individuelle Beziehung der Gestalter zu ihrem Garten bzw. kleinen Park als „Wohlfühloase“ wider. Es muss nicht immer ein alle Kontinente repräsentierender Botanischer Garten sein, sondern die (gelungene) „Synthese aus Natur und Design“, ein „Intimer Ort mit Weitblick“ oder „Harmonie aus Struktur und Dynamik“ können genauso reiz- voll sein. Dabei stehen weniger ökologische Parameter oder die Biodiversität im Fokus, vielmehr die kreativen Möglichkeiten, mit einer geeigneten Auswahl an Garten- und Parkgewächsen ein neues, individuelles Miniökosystem zu gestalten. Obwohl Gartenanlagen, insbesondere die von kommerziell orientierten Garten- und Landschaftsarchitekten, einer unvermeidbaren anthropologischen Überformung unterliegen (also künstlich konstruiert sind), bieten sie viele Chancen für kleine Fluchten aus dem Alltag. Sie können bzw. sollen als Anregung für die Gestaltung eines eigenen Gartens dienen.

$\mathrm{Zu}$ jeder Anlage erfährt der Leser im Vorspann diejenigen Fakten, die ihr das typische Gepräge verleihen, bevor die schnörkellose, detaillierte Beschreibung mit je einem Gartenplan folgt. Auch die Gestalter kommen stets zu Wort. Formalia wie Adresse, Kontaktmöglichkeit sowie eine Artenliste der im Buch genannten Pflanzen bilden den Schluss des Werkes.

Wer nicht gleich eine Gartenerkundungstour durch die Bundesländern bzw. die Schweizer Gärten machen möchte, wird sicher auch in seinem „Einzugsbereich“ einen der „Gärten des Jahres 2017 “ finden.

\title{
Anschriften der Autorinnen und Autoren
}

Angelika \& Heinz Baum, Klettenberggürtel 13, 50939 Köln, E-Mail: a.u.h.baum@web.de

Theodor C. H. Cole, Ziegelgasse 20, 69117 Heidelberg, E-Mail: tchcole@gmx.de

Dr. Veit Martin Dörken, Universität Konstanz, Fachbereich Biologie, Universitätsstraße 10, 78457 Konstanz,

E-Mail: veit.doerken@uni-konstanz.de

Dr. GÜNter Gerlach, Botanischer Garten München-Nymphenburg, Staatliche Naturwissenschaftliche Sammlungen Bayerns (SNSB), Menzinger Str. 61, 80638 München, E-Mail: gerlach@extern.lrz-muenchen.de
Rolf Goetz, Rudolstädter Str. 121, 10713 Berlin, E-Mail: rolfgoetz@arcor.de

Annette Höggemeier, Botanischer Garten, Ruhr-Universität Bochum, Universitätsstraße 150, 44780 Bochum

Bernd Margenburg, Auf der Klause 5, 59192 Bergkamen, E-Mail: bernd@mgorch.de

Volker Unterladstetter, Gellerstr. 45, 50733 Köln, E-Mail: volker.unterladstetter@botanik-bochum.de

Prof. I.R. Dr. Anton Weber, Department für Botanik und Biodiversitätsforschung, Universität Wien, Rennweg 14, A-1030 Wien, E-Mail: anton.weber@univie.ac.at

Nicht Genannte gehören dem Palmengarten an. 